\title{
Understanding the influence of a cognitively demanding task on motor response times and subjective mental fatigue/boredom
}

\author{
CHRIS THOMPSON $N^{1,2}$ | JOB FRANSEN² | ADAM BEAVAN $1,2,3$ | SABRINA SKORSKI'1 | AARON COUTTS'2 | TIM MEYER ${ }^{1}$
}

1 Institute of Sport and Preventive Medicine, Saarland University, Saarbrücken, Germany.

2 Sport and Exercise Discipline Group, Faculty of Health, University of Technology Sydney, Moore Park, Australia.

${ }^{3}$ German Football Association (Deutscher Fußball-Bund; DFB), Frankfurt, Germany.

Correspondence to: Chris Thompson. Saarland University - Institute of Sports and Preventive Medicine, Saarland University, Building 8.2, 66123 Saarbrücken, Germany.

University of Technology, Sydney - Moore Park Rd \& Driver Ave, Moore Park NSW 2021, Australia. +49 (0) 68130270413.

email: chris.thompson@uni.saarland.de

https://doi.org/10.20338/bjmb.v14i01.167

\begin{abstract}
HIGHLIGHTS
- The modified Stroop task is the most common method of inducing mental fatigue, yet may not be ecologically valid.

- The aim of the study was to investigate the influence of the modified Stroop task on mental fatigue, boredom and motor response times.

- This study has demonstrated that exposure to the modified Stroop task results in increased subjective mental fatigue and boredom; however there were no significant differences in motor task performance.

- The study also found that a short rest period (5-min) did not elicit a full subjective recovery from either parameter.
\end{abstract}

$\begin{array}{ll}\text { ABBREVIATIONS } \\ \text { AIC } & \text { Akaike's Information Criterion } \\ \text { AU } & \text { arbitrary units } \\ \text { CRTT } & \text { choice reaction time task } \\ \text { POST } & \text { post-task } \\ \text { POST-5 } & \text { five minute rest period } \\ \text { PRE } & \text { pre-task } \\ \text { VAS } & \text { visual analogue scale }\end{array}$

PUBLICATION DATA

Received 18022020

Accepted 20032020

Published 01042020

\begin{abstract}
BACKGROUND: Several methods are used to induce mental fatigue; predominantly the modified Stroop task, which arguably has little relation to daily lifestyle tasks.

AIM: To investigate the influence of the modified Stroop task on mental fatigue, boredom and motor response times.

METHOD: 15 subjects ( $24.3 \pm 2.3$ years) completed a 30 -min modified Stroop task (control condition: 30 -min reading) and completed PRE, POST and POST 5-min subjective ratings of mental fatigue, mental effort and boredom. Participants' ability to use congruent and inhibit incongruent precues in a choice reaction time task (CRTT) was measured pre- and post- Stroop and control.

RESULTS: Significantly higher subjective ratings of pre-post condition mental fatigue (pre: $2.43 \pm 1.31 \mathrm{AU}$, post: $6.42 \pm 2.08 \mathrm{AU}, \mathrm{p}<0.01$ ) and boredom (PRE: $1.65 \pm 1.49 \mathrm{AU}, \mathrm{POST}: 5.03 \pm 2.92 \mathrm{AU}, \mathrm{p}<0.01$ ) were reported following the modified Stroop task condition compared to the control condition mental fatigue (PRE: 1.62 $\pm 1.17 \mathrm{AU}, \mathrm{POST}$ : $2.10 \pm 0.92 \mathrm{AU}, p>0.05$ ) and boredom (PRE: $1.94 \pm 1.52 \mathrm{AU}, \mathrm{POST}: 3.32 \pm 1.66 \mathrm{AU}, \mathrm{p}>0.05$ ). No significant differences were found between conditions for the extent to which participants' response times were affected by congruent $(p=0.481)$ or incongruent $(p=0.225)$ precues.

CONCLUSION: Future research must adopt cognitive activities with higher contextual interference for greater ecological validity, and elucidate the impact of rest on recovery from mental fatigue.
\end{abstract}

KEYWORDS: Mental Fatigue | Boredom | Stroop

\section{INTRODUCTION}

Mental fatigue is a psychobiological state, characterised by feelings of tiredness, a lack of energy and reduced motivation that is induced by prolonged periods of demanding cognitive activity ${ }^{1,2}$. To induce mental fatigue, previous studies have used a range of cognitively demanding tasks. These include (but are not limited to) a 90-min AX-Continuous Performance Task $^{3,4,5}$ a 60-min Go/NoGo Task ${ }^{6}$, a 2-hour Eriksen Flanker test ${ }^{7,8}$, a blocked 4 x 10-min Sustained Attention to Response Task ${ }^{9}$, and in many recent studies, a 30-min modified Stroop task ${ }^{2,10}$. The modified Stroop task is a response inhibition/sustained attention task that elevates the activation of the anterior cingulate cortex, which appears to play a significant role in the onset of mental fatigue ${ }^{11}$. It has been theorised that following prolonged exposure to a

\begin{tabular}{l|l|l|l|ll}
\hline Thompson et al. & 2020 & VOL.14 & N.1 & https://doi.org/10.20338/bjmb.v14i01.167
\end{tabular}


Brazilian Journal of Motor Behavior

cognitively demanding task such as the modified Stroop task, mental fatigue results from a reduction in the transmission of dopamine to the striatum and anterior cingulate cortex 8,12 . Based on the current evidence, the use of the modified Stroop task can be regarded as a feasible activity to generally induce mental fatigue.

However, a limitation of the modified Stroop task is that it may not be representative of how mental fatigue is experienced in real life settings ${ }^{13}$. Indeed, this task is relevant in a laboratory setting to create a transient state of mental fatigue, but a driver would not complete a modified Stroop task before entering a car, nor would an athlete before a competition, making the practical validity of using a modified Stroop task questionable in high contextual situations. The repetitive nature of continually solving the same task problem may become monotonous and evoke lower levels of contextual interference than real life tasks such as driving, military activity and sport. Lelis-Torres et al. (2017) ${ }^{14}$ recently compared the task engagement associated with high and low contextual interference practice schedules (a key-pressing task with two goals: learning the relative timing dimension and learning the absolute timing dimension) and found that greater cognitive effort was more closely associated with high contextual interference than with low contextual interference practice. It has also been proposed that if a task is perceived to be repetitive, meaningless and does not challenge the subject, it may result in boredom ${ }^{15}$. Pattyn et al. (2008) $)^{15}$ explain an "underload" and "overload" performance hypothesis, where a cognitive task with an "underload" (i.e. low cognitive demand) is subjectively associated with mind wandering (boredom), whilst an "overload" task consists of a subjectively high cognitive demand when completing the NASA Task Load Index test (mental fatigue). It is plausible that the modified Stroop task may be regarded as boring by participants, but it remains unknown if impaired performance levels are partly caused by boredom due to low intrinsic motivation to engage in the task, rather than the demands of the task itself ${ }^{13,16}$. Despite the widespread use of a modified Stroop task as a means of inducing mental fatigue in the literature ${ }^{2,10}$, its difference or independence to boredom is yet to be investigated.

Furthermore, recent literature has suggested the need to supplement subjective perceptions of mental fatigue with more objectively quantifiable assessments ${ }^{13}$. One solution could be to assess the effect of mental fatigue on motor response times. Motor response times represent the ability of an individual to detect relevant information sources, process that information and use it to generate an appropriate movement response. Indeed, mental fatigue has been shown to impair attention ${ }^{17}$ and reaction times ${ }^{1}$. Moreover, response times represent the difference between the moment a stimulus appears and the subsequent execution of a motor response ${ }^{18}$. It is suggested that response time paradigms may be able to detect inefficiencies of the motor control system as a result of fatigue, including mental fatigue ${ }^{6,19}$. However, simple or even choice response time tasks also bear little relevance to performance in cognitively demanding activities such as driving or sport. Alongside being able to respond rapidly to a single stimulus (i.e. simple reaction time) or deciding between different responses based on a variety of stimuli (i.e. choice reaction time), such populations need to demonstrate fast and accurate decision making that includes the ability to use advanced information or suppress irrelevant stimuli. For example, a soccer player may decide to pass the ball to an available teammate but choose not to when observing their teammate is suddenly covered by a defender or another teammate is in a better position to receive the ball.

Limited evidence is also available on the amount of rest required to overcome subjective feelings of mental fatigue. A break (and not necessarily sleep) from a mentally fatiguing task will

\begin{tabular}{l|l|l|l|ll}
\hline Thompson et al. & 2020 & VOL.14 & N.1 & https://doi.org/10.20338/bjmb.v14i01.167
\end{tabular}


Brazilian Journal of Motor Behavior

result in some recovery ${ }^{20}$, but the duration of that break is unknown. In many sporting (e.g. timeouts and half-time periods) and occupational (rest periods from driving or factory duties) tasks, rest periods may be brief, and the amount of mental recovery (i.e. change in mental fatigue) sustained from these periods are unknown. Understanding the timing and duration of a break required to diminish the effects of mental fatigue is a crucial step to further understand the relationship between mental fatigue and motor performance, as it may help mitigate the potentially negative effects of mental fatigue on performance. Therefore, the aim of the current study was threefold. First, to investigate the effects of a modified Stroop task on participants' ability to use precued information in a choice response time task in which participants execute or suppress a motor response based on a congruent or incongruent precue preceding the stimulus. The secondary aim of the study was to understand the relationship between measures of mental fatigue, mental effort and boredom to understand whether the modified Stroop task represents an appropriate method to induce mental fatigue. The third aim of the study was to measure the impact of rest on recovery from mental fatigue and boredom. It was hypothesised that 1) a 30-min modified Stroop task would negatively affect the ability for participants to inhibit a motor response, 2) a 30-min modified Stroop task would significantly increase subjective ratings of mental fatigue, mental effort and boredom, and 3) an acute rest period (5-min) from cognitive activity would be sufficient to elicit significant reductions in subjective ratings of mental fatigue and boredom.

\section{METHODS}

\section{Participants}

Fifteen healthy adults $(24.3 \pm 2.3$ years) with normal or corrected to normal vision volunteered to participate in the study. Ethical approval for all procedures was attained from the Saarland University Ethics Committee under the Declaration of Helsinki.

\section{Procedures}

The study outline consisted of two separate protocols completed in a randomised counterbalanced order. Participants were informed that they would be completing a computer task and a reading task on two separate occasions, four weeks apart, in the same location at the same time of day. Participants were also told to refrain from consumption of caffeine for 24-hours before the study. Following this, participants provided their informed consent to participate. In both conditions, participants were placed in a rigid chair facing a desktop table containing a laptop computer and a customised four button controller. The controller was placed between the computer and the participant, precisely $8 \mathrm{~cm}$ from the edge of the computer. The dominant hand of the participant was placed flat on the table $3 \mathrm{~cm}$ away from the controller system, and the non-dominant hand was situated either on their thigh or the table. The familiarisation protocol consisted of a brief (12 trials) congruent version of the choice reaction time task (CRTT), a 60 s trial of the modified Stroop and a description of the reading task.

A pre-task (PRE) visual analogue scale (VAS) for mental fatigue and boredom were then completed, followed by the first CRTT trial. Next, participants were exposed to 30-min of either the modified Stroop task or the magazine reading condition. The test was completed with the researcher seated two metres behind the participant to ensure study compliance. Immediately post-task (POST), participants again completed VAS for mental fatigue, boredom and mental

\begin{tabular}{l|l|l|l|ll}
\hline Thompson et al. & 2020 & VOL.14 & N.1 & https://doi.org/10.20338/bjmb.v14i01.167
\end{tabular}


Brazilian Journal of Motor Behavior

effort, which corresponded to the amount of perceived mental effort that was required to complete the preceding task. The CRTT was then immediately completed for a second time, followed by a five minute rest period where the participant sat alone in the testing room with the instructions to relax and avoid any task engagement. To facilitate this, personal items (i.e. mobile phones, laptops, mobile phones) were securely stored outside of the testing room during the test. Finally, participants completed a final VAS for mental fatigue and boredom immediately following the five minute rest period (POST-5).

\section{Response Inhibition}

The response inhibition task (Unity, Version 5.4.0f3, 2016) consisted of 24 trials grouped into 12 congruent and 12 incongruent trials on a 14.3" laptop screen, lasting a total of three minutes. Across all trials, a three second countdown was followed by the appearance of four white circles with a black outline (all of which consist of a diameter of 512 pixels and an edge width of 5 pixels) presented in a horizontal position. Following a randomised two - four second delay, one of the four circles turned yellow. The participant was required to accurately press the button on the controller (Lioncast, Berlin, Germany) which corresponded to the yellow circle as quickly as possible. The precue in the congruent trials was a small black dot (26 pixels) which appeared in a white circle (512 pixels) $86 \mathrm{~ms}$ before it turned yellow. Conversely in the incongruent trials, the same black dot acted as a decoy by appearing in a white circle $86 \mathrm{~ms}$ before an opposing circle turned yellow. In both trials, the precue was presented on the screen for a duration of $43 \mathrm{~ms}$ according to the procedures outlined in one of the experimental conditions of an implicit precue paradigm used by Fransen et al. (in press) ${ }^{21}$, but adapted to include a measure of response inhibition.

\section{Modified Stroop task}

In the modified Stroop task, four words ("blue", "yellow", "red", "green") repeatedly appeared on a Windows Powerpoint Presentation document screen in a randomised fashion. The correct answer in the trial will be the colour of the ink (blue, yellow, red, green), and not the word. However, to increase task difficulty, any word presented in red ink the correct response corresponds to the meaning of the word. The test was translated into German, the first language of the participants. Each participant completed 900 trials, each separated by $2000 \mathrm{~ms}$. All trials required a verbal response to the researcher, who was seated two metres behind the participant to avoid acting as a visual distraction during the test.

\section{Control Condition}

In the control condition, participants were presented with a selection of four magazines of varying topics to read (sport, cars, fashion and lifestyle). They were instructed to read any of the chosen magazines for a period of 30-min whilst seated in front of the lead researcher. The choice of magazines was influenced by previous pilot testing.

\section{Visual analogue scale}

A visual analogue scale (VAS) was used to measure three subjective parameters; 1) boredom ("Please state your current level of boredom"), 2) mental fatigue ("Please state your current level of mental fatigue") and 3) mental effort ("Please state the mental effort of the task you have just completed"). On a $100 \mathrm{~mm}$ horizontal line scale, the answer selection ranged from

\begin{tabular}{l|l|l|l|ll}
\hline Thompson et al. & 2020 & VOL.14 & N.1 & https://doi.org/10.20338/bjmb.v14i01.167
\end{tabular}


"none at all" to "maximal". Using an independent paper version for each time point (to avoid participants comparing subjective measures to previous time points), participants were instructed to draw a vertical line over a selected area of the scale to select their subjective rating of each parameter, which was later measured by the lead researcher to create arbitrary units $(A U)$.

\section{Statistical analysis}

To investigate the effect of the experimental condition (i.e. the reading task (control) or the modified Stroop task and timing of the measurement (PRE, POST \& POST-5) on both cognitive (mental fatigue, mental effort and boredom) and response times (congruent and incongruent task performance), a series of linear mixed models was developed. A stepwise approach was used in which additional predictors were added to the model with each step, and model fit was evaluated using the Akaike's Information Criterion (AIC), observation of increases in degrees of freedom, a -2 log-likelihood ratio test and the normal distribution of the models' residuals. The cognitive variables and response times were entered as response variables in each of the four linear mixed models. The experimental condition and the time of measurements (fixed factors) were entered as predictor variables in addition to each participant's unique identifier (random factor) to account for the random variance associated with the clustering of participants' repeated measures within each participant. Prior to the analysis, pre-modelling assumption checks (i.e. linearity of relationships, homogeneity of variance) were carried out. Following the analysis, the appropriateness of each model was analysed through the normality of the distribution of model residuals using visual inspection through boxplots and a Shapiro-Wilk test. The significance level for the -2 log-likelihood ratio tests was set at $p<0.05$, and an estimate precision was provided using Wald-based 95\% confidence intervals. In order to understand if an association existed between the various outcomes due to the Stroop test, Pearson correlations were conducted on the delta change between each variable.

\section{RESULTS}

The best fit was achieved using a random intercepts model (participant ID) and through the introduction of a time*experimental condition interaction effect to explain the variance in mental fatigue and boredom. However, no significant predictors of congruent and incongruent response times were observed in this study $(p>0.05)$. As mental effort was only recorded during the post-test, a random intercept model with the experimental condition as the only fixed effect best explained the variance in mental effort $(\mathrm{AIC}=112.42, \mathrm{df}=1-, 4, \mathrm{p}=<0.001$, conditional explained variance $=75 \%$ ). A random intercepts model that incorporated both the condition and time main effects as well as the condition ${ }^{\star}$ time interaction effect best explained the variance in subjective ratings of mental fatigue and boredom (mental fatigue: $\mathrm{AIC}=326.39$, $\mathrm{df}=2,8, p=$ $<0.001$, conditional explained variance $=66 \%$; boredom: $\mathrm{AIC}=371.14, \mathrm{df}=3,8, \mathrm{p}=<0.021$, conditional explained variance $=54 \%$ ). All model parameters organised by dependent variable can be found in Table 1.

As shown in Figure 1, a significant condition*time interaction effect $(p<0.001)$ was observed in ratings of mental fatigue. Mental fatigue values increased from PRE (2.43 \pm 1.31 $\mathrm{AU})$ to POST $(6.42 \pm 2.08 \mathrm{AU} ; \mathrm{p}<0.01)$ in the modified Stroop task condition. Furthermore, despite the POST-5 mental fatigue values decreasing $(5.26 \pm 1.49 \mathrm{AU} ; p<0.01)$ from the POST

\begin{tabular}{l|l|l|l|ll}
\hline Thompson et al. & 2020 & VOL.14 & N.1 & https://doi.org/10.20338/bjmb.v14i01.167 & 37 of 45 \\
& & & &
\end{tabular}


Brazilian Journal of Motor Behavior

\section{Research Article}

test values, they still remained higher $(p<0.01)$ compared to the PRE test values within the Stroop task condition. Contrastingly no observable differences $(p>0.05)$ were recorded in the control condition across the PRE, POST or POST-5 time points (see Table 1). Pearson correlations were conducted between the delta change from pre to post Stroop test between the variables response inhibition, mental fatigue and boredom. The results demonstrated that a moderate positive correlation between increases in mental fatigue and boredom ratings $(r=$ $0.46, p=0.09$ ) existed although failed to reach significance. Furthermore, very low correlations existed between the delta change of response inhibition and ratings of mental fatigue $(r=0.08$, $p=0.80)$ or ratings of boredom $(r=-0.17, p=0.55)$.

Subjective rating of boredom followed a similar trend to mental fatigue for both conditions, and a significant condition*time interaction effect $(p=0.021)$ was also observed. In the modified Stroop condition, ratings of boredom increased from PRE (1.65 $\pm 1.49 \mathrm{AU})$ to POST $(5.03 \pm 2.92 \mathrm{AU} ; p<0.01)$. Aligned with the trends of mental fatigue ratings, POST-5 boredom values $(3.85 \pm 2.07 \mathrm{AU})$ also decrease from POST values, but remained higher $(p<0.01)$ from the initial PRE test values. Ratings of boredom were also similar to the ratings of mental fatigue in the control condition, where no significant differences $(p>0.05)$ were observed across PRE, POST, and POST- 5 time points.

Furthermore, a conditional main effect $(p<0.001)$ was observed for mental effort scores recorded at POST, as participants reported higher ratings of mental effort in the modified Stroop condition (6.67 $\pm 1.72 \mathrm{AU})$ compared to the control condition (1.87 $\pm 1.05 \mathrm{AU})$. Lastly, there were no differences in congruent or incongruent response times between the modified Stroop task and control conditions (see Table 1). The estimated marginal means, standard errors and associated $95 \%$ confidence intervals derived from the best fitting models for each dependent variable are presented in Table 2.

Figure 1. Pre and post VAS ratings of subjective mental fatigue and boredom.

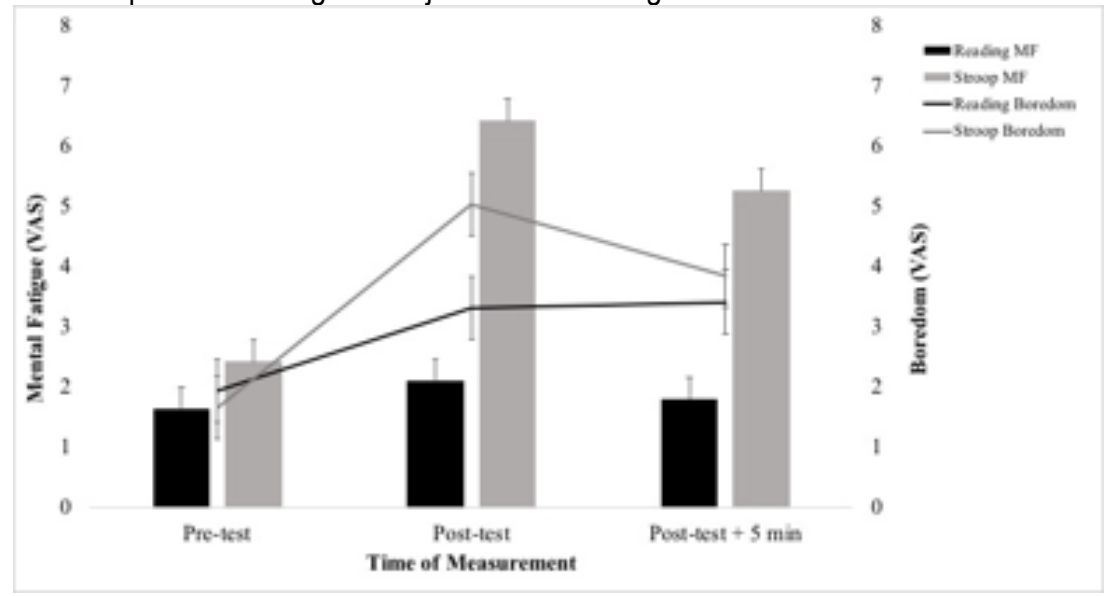


Brazilian Journal of Motor Behavior

Table 1 - Models that explain the effect of condition and time of measurement on participants' cognitive and physical variables.

\begin{tabular}{|c|c|c|c|c|c|c|}
\hline & $\mathrm{AIC}$ & $p(-2$ log likelihood ratio test) & $\mathrm{Chi}^{2}$ & df & $\mathrm{R}^{2}$ fixed only (\%) & Conditional $\mathrm{R}^{2}$ random + fixed (\%) \\
\hline \multicolumn{7}{|l|}{ Congruent } \\
\hline \multicolumn{7}{|c|}{ Final Model: Congruent $\sim 1+(1|| \mathrm{D})$} \\
\hline Condition main effect & -210.18 & 0.733 & 0.116 & 1,4 & $<0.1$ & 64 \\
\hline Time main effect & -210.48 & 0.519 & 0.416 & 1,4 & 0.2 & 64 \\
\hline Time + Condition main effect & -208.59 & 0.766 & 0.533 & 2,5 & 0.3 & 64 \\
\hline Interaction effect & -208.53 & 0.481 & 2.468 & 3,6 & 1.4 & 65 \\
\hline \multicolumn{7}{|l|}{ Incongruent } \\
\hline \multicolumn{7}{|c|}{ Final Model: Incongruent $\sim 1+(1 \mid$ ID $)$} \\
\hline Condition main effect & -220.48 & 0.611 & 0.259 & 1,4 & 0.1 & 78 \\
\hline Time main effect & -220.26 & 0.848 & 0.037 & 1,4 & $<0.1$ & 78 \\
\hline Time + Condition main effect & -218.52 & 0.862 & 0.296 & 2,5 & 0.1 & 77 \\
\hline Interaction effect & -220.28 & 0.225 & 4.061 & 3,6 & 1.4 & 78 \\
\hline \multicolumn{7}{|l|}{ Mental fatigue } \\
\hline \multicolumn{7}{|c|}{ Final Model: Mental fatigue $\sim$ Time ${ }^{\star} \operatorname{Exp}$ Cond $+(1 \mid$ ID $)$} \\
\hline Condition main effect & 370.31 & $<0.001$ & 43.663 & 1,4 & 38 & 38 \\
\hline Time + Condition main effect & 346.80 & $<0.001$ & 27.514 & 2,6 & 54 & 54 \\
\hline Interaction effect* & 326.39 & $<0.001$ & 24.412 & 2,8 & 64 & 66 \\
\hline \multicolumn{7}{|l|}{ Mental effort } \\
\hline \multicolumn{7}{|c|}{ Final Model: Mental effort $\sim$ ExpCond + (1|ID) } \\
\hline Conditional main effect* & 112.42 & $<0.001$ & 41.801 & 1,4 & 75 & 75 \\
\hline \multicolumn{7}{|l|}{ Boredom } \\
\hline \multicolumn{7}{|c|}{ Final Model: Boredom Time ExpCond + (1|ID) } \\
\hline Condition main effect & 399.52 & 0.134 & 2.248 & 1,4 & 2 & 29 \\
\hline Time main effect & 374.87 & $<0.001$ & 28.891 & 2,5 & 19 & 50 \\
\hline Time + Condition main effect & 373.54 & 0.068 & 3.328 & 1,6 & 21 & 51 \\
\hline Interaction effect* & 371.14 & 0.021 & 9.7301 & 3,8 & 24 & 54 \\
\hline
\end{tabular}

Note: * indicates the best fitting model based on AIC value and -2log-likelihood ratio test. ID = individual participant identification number, Time $=$ time of measurement (i.e. pre-test, posttest or post-test +5 minutes break), ExpCond $=$ experimental condition (i.e. Control or Stroop).

\begin{tabular}{l|l|l|l|lc}
\hline Thompson et al. & 2020 & VOL.14 & N.1 & https://doi.org/10.20338/bjmb.v14i01.167 & 39 of 45
\end{tabular}


Brazilian Journal of Motor Behavior

Table 2 - Least square means, 95\% confidence intervals (Cl), standard error (Std. Error), t-values and random effect parameters from four linear mixed models investigating the effects of condition and time of measurement on participants' cognitive and physical variables.

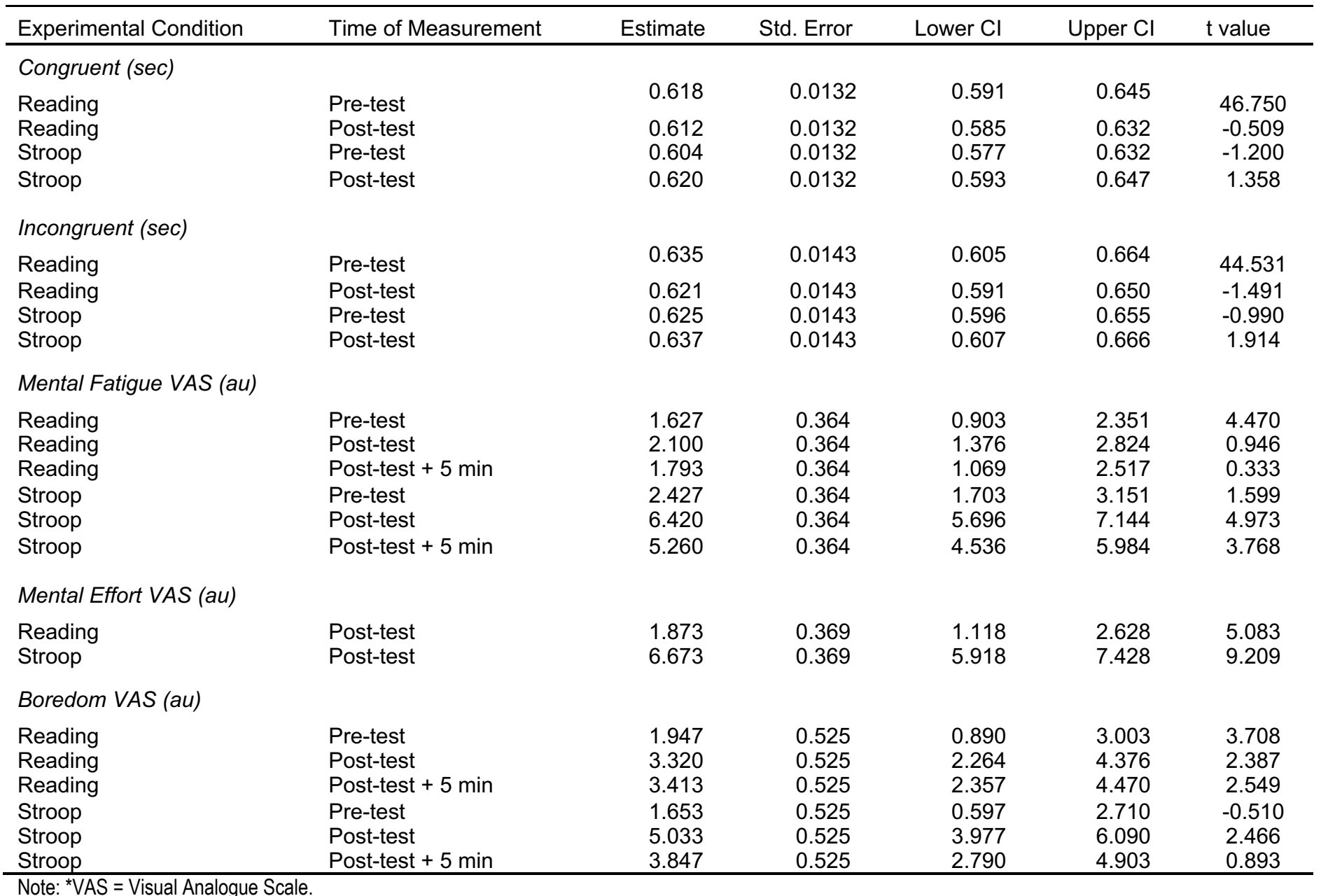

Note: ${ }^{*}$ AS = Visual Analogue Scale.

\begin{tabular}{l|l|l|l|ll}
\hline Thompson et al. & 2020 & VOL.14 & N.1 & https://doi.org/10.20338/bjmb.v14i01.167
\end{tabular}




\section{DISCUSSION}

For a number of years, many different cognitive tasks have been used to induce mental fatigue $^{3-9}$, with the 30-min modified Stroop task being the predominant method ${ }^{2,10}$. This has recently been challenged for its low contextual interference and irrelevance to daily tasks ${ }^{13}$, which may lead to low intrinsic motivation to engage in the activity and ultimately subjective boredom. Furthermore, the amount of time required to rest from mentally fatiguing tasks is yet to be investigated in the literature. Our study has demonstrated that in comparison to a control condition (reading magazines), 30-min exposure to the modified Stroop resulted in significantly greater subjective mental fatigue, mental effort and boredom, but did not significantly impair motor task performance. Moreover, a 5-min rest period was not enough to provide a full subjective recovery of mental fatigue and boredom. The results of the current study support the applicability of the modified Stroop task in inducing mental fatigue in laboratory settings, but also provides new evidence to support the presence of subjective boredom during the task. Furthermore, evidence has been presented for the impact of acute rest from mentally fatiguing activity, which provides future suggestions for inducing mental fatigue in subsequent research.

The primary finding in the current study was the greater subjective boredom reported following the 30-min modified Stroop task condition in comparison to the control task. These findings support the argument that the modified Stroop task is boring, and this boredom may subsequently influence the perception of mental fatigue. Ultimately the modified Stroop task may not be a relevant task to induce mental fatigue due to its irrelevance to many real-world tasks ${ }^{13}$. It has been suggested that boredom is linked to monotony ${ }^{22}$, and that impaired task performance is associated with low intrinsic attractiveness of the task ${ }^{16}$. In the present study, the 30-min modified Stroop task is likely to have been met with low intrinsic motivation and task engagement due to its irrelevance to daily activities and no perceived reward. The nature of the task is highly repetitive with little variation and low contextual interference. Indeed, low contextual interference tasks provide a lesser cognitive engagement than high contextual interference tasks ${ }^{14}$, and likely provides a cognitive "underload"15. Here it was theorised that compared to "overload" tasks (i.e. high cognitive engagement), an "underload" task provides a reduced cognitive challenge and is associated with mind wandering (i.e. boredom) ${ }^{15}$. This evidence suggests that whilst the modified Stroop task may bear a resemblance to some realworld tasks with prolonged vigilance and low cognitive engagement (i.e. repetitive factory or office based tasks), more dynamic tasks (e.g. sport, military activity) require a more ecologically valid protocol to induce mental fatigue in future studies. To develop such protocols, future research must further understand real-world (such as lifestyle or task specific) cognitive demands experienced by individuals in dynamic task performers.

Despite significant increases in subjective mental fatigue and boredom, congruent and incongruent response times were unaffected by exposure to a 30-min modified Stroop task. The results are contrary to the hypothesis and somewhat surprising given the available literature. It has previously been reported that participants performing a 60-min Go/NoGo recorded significantly greater reaction times, number of errors, and mental fatigue scores with time spent on the task ${ }^{6}$. These results demonstrate a decrement in response inhibition performance, along with an impairment of the intensity of response execution. In similar work, participants performing a 2-hour Eriksen Flanker test showed a significantly greater decline in reaction times

\begin{tabular}{l|l|l|l|ll}
\hline Thompson et al. & 2020 & VOL.14 & N.1 & https://doi.org/10.20338/bjmb.v14i01.167
\end{tabular}


Brazilian Journal of Motor Behavior

during the final 30 -min period in comparison to the initial 90-min of the task. Additionally, when exposed to a 30-min modified Stroop task, Smith et al. (2016) ${ }^{23}$ demonstrated very likely lower decision making accuracy and likely higher response times in a soccer specific soccer decision making test in comparison to a control condition (reading magazines). There may be multiple explanations as to why these results differed to the current study. Firstly, the 60-min Go/NoGo task and 2-hour Eriksen Flanker test used by Kato et al. $(2009)^{6}$ and Lorist, Boksem \& Ridderinkhof $(2005)^{8}$ were significantly greater in the duration than the 30-min modified Stroop task used in the current study, which suggests that time on task correlates with decrement in cognitive function. In addition, Smith et al. (2016) ${ }^{23}$ used a soccer-specific task in a sample of soccer players, thus using a sport-specific stimulus may complicate the comparison of the results of these two studies. However, the fact that congruent and incongruent response times remained unaffected in the current study, increases in subjective ratings of mental fatigue and boredom may be due to the 30-min modified Stroop task's inability to elicit true mental fatigue or the ability to use implicit information to inform action is unaffected by mental fatigue.

The present findings show that a five minute rest period following the 30-min modified Stroop task significantly reduced subjective ratings of mental fatigue and boredom but did not fully dissipate to baseline levels after the rest period. Despite the inability to fully recover, the study shows that even a five minute period was enough to significantly reduce subjective mental fatigue. This suggests that mental fatigue is transient and stimulus dependent, which conflicts the severity of mental fatigue on task performance as theorised in previous research ${ }^{24,25}$. However, a limitation of the study is that each subject participated in the protocol at varying times (but consistent within each subject) of the day (morning to early evening).Indeed, the ability to subjectively recover from the 30-min modified Stroop may have been influenced by this variability and future study designs interested in the acute effects of mental fatigue on performance should aim to assess each participant at the same time of the day. Other considerations in relation to this are the present workload experienced by the participants, as well as sleep duration, which was not measured in the study. Additionally, whilst periods of rest or time away from cognitive tasks have been previously advocated as methods to reduce feelings of mental fatigue ${ }^{19,26}$, the timing and dosing of recovery following mentally fatiguing tasks remains unknown. However, it must be emphasised that clearly interpreting post task subjective measurements of mental fatigue and boredom is challenging. Participants in the current study were required to sit alone with no stimulation (i.e. in silence with no mobile phone/laptop access), which in longer periods may even further exacerbate subjective feelings of mental fatigue and boredom, which has been likened to a high level of frustration ${ }^{21}$.

\section{CONCLUSION}

In summary, this study demonstrated that despite greater subjective mental fatigue, boredom and mental effort in the modified Stroop condition, participants' ability to use congruent and suppress incongruent precues was maintained in comparison to a control condition. Moreover, a five minute break reduced subjective mental fatigue and boredom in the 30-min modified Stroop condition, but not significantly. The authors suggest that the modified Stroop task may not be a "one size fits all" approach to inducing mental fatigue when considering the level of contextual interference of a particular task. To allow for further understanding of how such complex cognitive tasks are influenced by mental fatigue, it is recommended that future

\begin{tabular}{l|l|l|l|ll}
\hline Thompson et al. & 2020 & VOL.14 & N.1 & https://doi.org/10.20338/bjmb.v14i01.167
\end{tabular}


research understands real-life activities experienced by complex task performers. This will in turn create more ecologically valid protocols which bear a greater resemblance than the modified Stroop task.

\section{REFERENCES}

1. Boksem, M. A., Meijman, T. F., \& Lorist, M. M. (2005). Effects of mental fatigue on attention: an ERP study. Cognitive Brain Research, 25(1), 107-116

2. Van Cutsem, J., Marcora, S., De Pauw, K., Bailey, S., Meeusen, R., \& Roelands, B. (2017). The effects of mental fatigue on physical performance: a systematic review. Sports Medicine, 47(8), 1569-1588.

3. Marcora, S. M., Staiano, W., \& Manning, V. (2009). Mental fatigue impairs physical performance in humans. Journal of Applied Physiology, 106(3), 857-864.

4. MacMahon, C., Schücker, L., Hagemann, N., \& Strauss, B. (2014). Cognitive fatigue effects of physical performance during running. Journal of Sport and Exercise Psychology, 36(4), 375381.

5. Smith, M. R., Marcora, S. M., \& Coutts, A. J. (2015). Mental Fatigue Impairs Intermittent Running Performance. Medicine and Science in Sports and Exercise, 47(8), 1682.

6. Kato, Y., Endo, H., \& Kizuka, T. (2009). Mental fatigue and impaired response processes: event-related brain potentials in a Go/NoGo task. International Journal of Psychophysiology, 72(2), 204-211.

7. Faber, L. G., Maurits, N. M., \& Lorist, M. M. (2012). Mental fatigue affects visual selective attention. PLOS ONE, 7(10), e48073.

8. Lorist, M. M., Boksem, M. A., \& Ridderinkhof, K. R. (2005). Impaired cognitive control and reduced cingulate activity during mental fatigue. Cognitive Brain Research, 24(2), 199-205.

9. Dillard, M. B., Warm, J. S., Funke, G. J., Funke, M. E., Finomore Jr, V. S., Matthews, G., Shaw, T.H., \& Parasuraman, R. (2014). The sustained attention to response task (SART) does not promote mindlessness during vigilance performance. Human Factors, 56(8), 13641379.

10. Smith, M. R., Thompson, C., Marcora, S. M., Skorski, S., Meyer, T., \& Coutts, A. J. (2018). Mental fatigue and soccer: Current knowledge and future directions. Sports Medicine, 1-8.

11. MacDonald, A. W., Cohen, J. D., Stenger, V. A., \& Carter, C. S. (2000). Dissociating the role of the dorsolateral prefrontal and anterior cingulate cortex in cognitive control. Science, 288(5472), 1835-1838.

12. Martin, K., Meeusen, R., Thompson, K. G., Keegan, R., \& Rattray, B. (2018). Mental fatigue impairs endurance performance: a physiological explanation. Sports Medicine, 1-11.

13. Thompson, C. J., Fransen, J., Skorski, S., Smith, M. R., Meyer, T., Barrett, S., \& Coutts, A. J. (2018). Mental fatigue in football: is it time to shift the goalposts? An evaluation of the current methodology. Sports Medicine, 1-7. 
14. Lelis-Torres, N., Ugrinowitsch, H., Apolinário-Souza, T., Benda, R. N., \& Lage, G. M. (2017). Task engagement and mental workload involved in variation and repetition of a motor skill. Scientific Reports, 7(1), 14764.

15. Pattyn, N., Neyt, X., Henderickx, D., \& Soetens, E. (2008). Psychophysiological investigation of vigilance decrement: Boredom or cognitive fatigue? Physiology \& Behavior, 93, 369-378.

16. Hockey, G. R. J. (2011). A motivational control theory of cognitive fatigue. Cognitive fatigue: Multidisciplinary Perspectives on Current Research and Future Applications, 167-87.

17. Chaudhuri, A., \& Behan, P. O. (2004). Fatigue in neurological disorders. The Lancet, 363(9413), 978-988.

18. Schmidt, R., \& Lee, T. (2013). Motor learning and performance, $5 E$ with web study guide: from principles to application. Human Kinetics. Page $22-23$.

19. Lorist, M. M., Bezdan, E., ten Caat, M., Span, M. M., Roerdink, J. B., \& Maurits, N. M. (2009). The influence of mental fatigue and motivation on neural network dynamics; an EEG coherence study. Brain Research, 1270, 95-106.

20. Balkin, T. J. and N. J. Wesensten (2011). Differentiation of sleepiness and mental fatigue effects. Cognitive fatigue: Multidisciplinary perspectives on current research and future applications. P. L. Ackerman. Washington, DC, American Psychological Association: 47-66.

21. Fransen, Novak, Turner, Tosi Rodrigues, Barela. Implicit precues are an affordable for action in a choice response time task. In press.

22. Hill, A. B., \& Perkins, R. E. (1985). Towards a model of boredom. British Journal of Psychology, 76(2), 235-240.

23. Smith, M. R., Zeuwts, L., Lenoir, M., Hens, N., De Jong, L. M., \& Coutts, A. J. (2016). Mental fatigue impairs soccer-specific decision-making skill. Journal of Sports Sciences, 34(14), 1297-1304.

24. Coutts, A. J. (2016). Fatigue in football: it's not a brainless task!. Journal of Sports Sciences, 34(14), 1296.

25. Walsh, V. (2014). Is sport the brain's biggest challenge?. Current Biology, 24(18), R859-R860.

26. Wesensten, N. J., G. Belenky, D. R. Thorne, M. A. Kautz and T. J. Balkin (2004). "Modafinil vs. caffeine: effects on fatigue during sleep deprivation." Aviation, Space, and Environmental Medicine 75(6): 520-525. 
Brazilian Journal of Motor Behavior

Citation: Thompson C, Fransen J, Beavan A, Skorski S, Coutts A, Meyer T. Understanding the influence of a cognitively demanding task on motor response times and subjective mental fatigue/boredom. BJMB. 2020: 14(1): 33-

45.

Editors: Dr Fabio Augusto Barbieri - São Paulo State University (UNESP), Bauru, SP, Brazil; Dr José Angelo Barela São Paulo State University (UNESP), Rio Claro, SP, Brazil; Dr Natalia Madalena Rinaldi - Federal University of Espírito Santo (UFES), Vitória, ES, Brazil.

Copyright:@ 2020 Thompson, Fransen, Beavan, Skorski, Coutts and Meyer and BJMB. This is an open-access article distributed under the terms of the Creative Commons Attribution-Non Commercial-No Derivatives 4.0 International License which permits unrestricted use, distribution, and reproduction in any medium, provided the original author and source are credited.

Funding: There was no funding for this study.

Competing interests: The authors have declared that no competing interests exist.

DOI: https://doi.org/10.20338/bjmb.v14i01.167 